\title{
GENETIC DIFFERENCES BETWEEN SACCHAROMYCES CARLSBERGENSIS AND S. CEREVISIAE II. RESTRICTION ENDONUCLEASE ANALYSIS OF GENES IN CHROMOSOME III
}

\author{
by \\ STEEN HOLMBERG
}

Department of Physiology, Carlsberg Laboratory

Gamle Carlsberg Vej 10, DK-2500 Copenhagen, Valby

Keywords: Brewers yeast, DNA hybridization, silent mating type loci, MAT, HML, HMR, HIS4, LEU2, SUP-RLI

\begin{abstract}
Chromosome III in a haploid Saccharomyces cerevisiae strain has been previously substituted for by its homeologue from S. carisbergensis. With this chromosome substitution line the two homeologous chromosomes were shown to undergo crossing over only in a limited region, and to differ in nucleotide sequence at the HIS4 locus. In the present study the S. carlsbergensis chromosome III was compared to its S. cerevisiae homeologue at several additional loci.

Cloned DNA from the S. cerevisiae loci $H M L$. HIS4, LEU2, MAT and SUP-RLI was used as hybridization probes in the analysis of nucleotide sequence homology at these loci and $H M R$. Virtually no differences were detected at SUP-RLI and $H M R$, located in the region where the two chromosomes recombine, whereas considerable differences were found in the non-recombining part. The data are consistent with the assumption that $H M L, M A T$ and $H M R$ of the $\mathrm{S}$. carlsbergensis chromosome are organized as in S. cerevisiae. Segments $\mathrm{X}$ and $\mathrm{Z} 1$, which are involved in mating type interconversion, were closely homologous to their $\mathrm{S}$. cerevisiae counterparts, whereas $\mathrm{Y} \alpha$ as well as sequences outside the $H M L$ and $M A T$ cassettes were substantially different.
\end{abstract}

\section{INTRODUCTION}

Single chromosomes can be transferred from one haploid nucleus to the other in karl x KARI heterokaryons of Saccharomyces cerevisiae $(8,24)$. Making use of this phenomenon, NiLsSON-TILLGREN et al. (25) isolated S. cerevisiae strains with an extra $\mathrm{S}$. carlsbergensis chromosome III from crosses between spore derived clo- nes of a $\mathrm{S}$. carlsbergensis brewing strain and haploid karl S. cerevisiae strains. During the study of the disomic strains a derivative which had lost the S. cerevisiae chromosome III was identified. The retained $\mathrm{S}$. carlsbergensis chromosome had a close functional homology to $S$. cerevisiae chromosome III. Genetic analysis (25), on the other

Abbreviations: $b p=$ base pair; $k b=$ kilobase pair 
S. Holmberg: S. carlsbergensis chromosome III

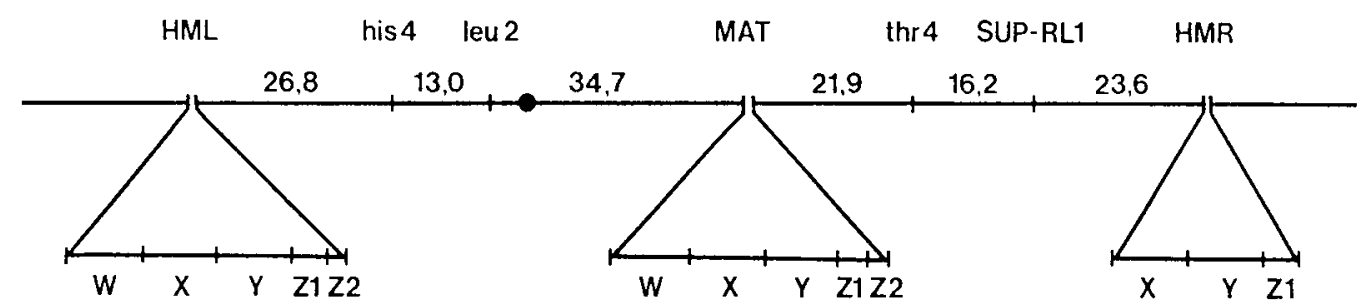

Figure 1. Map of relevant markers on chromosome III of S. cerevisiae.

The map distances, expressed in centimorgans, are from MORTIMER and SCHILD (20).

hand, revealed that the S. carlsbergensis chromosome III only recombined with its $\mathrm{S}$. cerevisiae counterpart in a limited region. Moreover, molecular analysis showed that the chromosome had a nucleotide sequence in the HIS4 region which differed from that of S. cerevisiae. Since several other genes located on chromosome III have been cloned the analysis can be extended to include $\operatorname{LEU} 2(27,30)$, coding for $\beta$-isopropylmalate dehydrogenase and $S U P-R L I$, a recessive lethal amber suppressor (26), as well as the mating type loci $M A T, H M L$ and $H M R(12,21)$. The genetic map for these gene loci is presented in Figure 1.

The mating type, $\mathbf{a}$ or $\alpha$, is determined by the $M A T$ locus which contains one of two unique wild type DNA sequences $(21,33)$. Haploid nuclei containing the $\mathrm{HO}$ (homothallism) gene switch mating type frequently by substituting Ya at MAT with $Y \alpha$ or vice versa. These substitutions require the presence of $\mathrm{Ya}$ or $\mathrm{Y} \alpha$, respectively, at the silent mating type loci, $H M L$ and $H M R$ (11). Most strains are $H M L \alpha H M R \mathbf{a}$, i.e. $H M L$ contains $\mathrm{Y} \alpha$ and $H M R$ contains $\mathrm{Ya}$, but strains with the reciprocal combination or with the same $\mathrm{Y}$ at both silent loci also exist. Similarities in the structure of $M A T$ and the two $H M$ loci ( $H M L$ and $H M R$ ) have been demonstrated by heteroduplex, restriction site mapping as well as DNA nucleotide sequence analysis $(2,21,33)$. At the center of each locus lies $Y$, the sequence specific for the mating type, consisting either of the 650 base pair (bp) long Ya or the 750 bp long Ya stretch. Characteristic sequences flanking $\mathrm{Y}$ are found at both silent loci as well as $M A T$. A $700 \mathrm{bp}$ region, $X$, to the left of $Y$, and a $230 \mathrm{bp}$ sequence, $Z 1$, to the right of $Y$, are common to all three loci. At each locus, the cluster of the $\mathrm{X}, \mathrm{Y}$ and $\mathrm{Zl}$ segments is called the cassette. Two further sequences, $\mathrm{W}$ and $\mathrm{Z} 2$, are present at $H M L$ and
$M A T$, but missing at $H M R$. W is 700 bp long and positioned left of $X$, whereas $Z 2$ comprises $90 \mathrm{bp}$ to the right of $\mathrm{Zl}$.

In the present study cloned DNA sequences from $H M L, H I S 4, L E U 2, M A T$ and SUP-RLI of $S$. cerevisiae were used as hybridization probes to analyse the structure of the corresponding genes in the S. carlsbergensis chromosome III. Consistent with the genetic recombination pattern the chromosome regions containing HML, HIS4, $L E U 2$ and $M A T$ were found to have nucleotide sequences differing from those of $\mathrm{S}$. cerevisiae while the segments including SUP-RLI and $H M R$ were closely related. The analysis of the mating type loci was carried out in greater detail using a subset of DNA restriction fragments derived from $H M L \alpha$ and $M A T \alpha$. A high degree of nucleotide sequence conservation is observed between $\mathrm{S}$. carlsbergensis and $\mathrm{S}$. cerevisiae cassettes of the mating type loci. The closest agreement is seen for the regions involved in the transposition of $\mathrm{Y}$ during switching of mating type.

\section{MATERIALS AND METHODS}

\subsection{Strains and recombinant molecules}

The following yeast strains were used as sources of DNA: Saccharomyces cerevisiae strain $\mathrm{S} 288 \mathrm{C}(M A T \alpha)$, S. carlsbergensis lager production strain 244 and strain C80-1253 (MATa), which is derived from the haploid strain K5-5A by substituting its chromosome III with a chromosome III from strain 244 (25).

The following plasmids were used as sources for DNA restriction endonuclease fragments: pYF91.2, a TC ${ }^{\mathrm{R}}$ derivative of pYF91 (30), containing the $L E U 2$ gene from $\mathrm{S}$. cerevisiae strain S288C on a 6 kilobase pair (kb) HindIII - BamHI fragment, was a gift from J. FRIESEN; pPM6 (26), containing a $1.1 \mathrm{~kb}$ BamHI - EcoRI frag- 
ment mapping $1.6 \mathrm{~kb}$ from $S U P-R L 1$ from strain Y4A, was a gift from M. OLson; pC503 (14), containing the HIS4 gene from the diploid S. cerevisiae strain A364A D5 $\mathrm{U}^{+} \mathrm{A}^{+}$on a $9.4 \mathrm{~kb}$ PstI fragment; pBR322 with a $6.1 \mathrm{~kb}$ HindIII insert containing $H M L \alpha$ from the haploid $\mathrm{S}$. cerevisiae strain XP8-10B $(21,23)$ and pBR322 with a $4.3 \mathrm{~kb}$ HindIII insert containing $M A T \alpha$ from strain XP8-10B $(21,23)$ were gifts from $\mathrm{K}$. TATCHELl.

\subsection{Media}

For yeast the growth medium was YPD ( $1 \%$ yeast extract, $2 \%$ peptone, $2 \%$ glucose). E. coli was grown in LB ( $1 \%$ tryptone, $0.5 \%$ yeast extract, $1 \% \mathrm{NaCl}, 0.1 \%$ glucose).

\subsection{DNA purification}

Supercoiled plasmid DNA from E. coli was isolated by a 50 times scaled up version of the alkaline-SDS procedure (3) and purified by centrifugation in $\mathrm{CsCl}$ /ethidium bromide gradients. The preparation of total yeast DNA has been described previously $(14,16)$.

\subsection{Enzymes, electrophoresis and purification of specific DNA fragments}

Restriction endonucleases and DNA polymerase I were purchased from Boehringer Mannheim Biochemicals and Bethesda Research Laboratories Inc. Gel electrophoresis of DNA (16) was performed in $0.7 \%$ or $2 \%$ agarose. Restriction fragments were purified from agarose by electroelution onto a dialysis membrane according to YANG et al. (36); after electroelution ethidium ions were removed with Dowex 50 and the DNA was precipitated in $0.3 \mathrm{M}$-sodium acetate with 2.5 volumes of $96 \%$ ethanol.

\subsection{In vitro labelling of DNA, Southern transfers and hybridization}

Purified restriction fragments were labelled in vitro with ${ }^{32} \mathrm{P}$-dTTP (Radiochemical Centre Amersham or New England Nuclear) by nick translation according to RIGBY et al. (28). DNA fragments of genomic yeast DNA distributed in agarose gels were transferred to a nitrocellulose filter (Millipore, HAWP 00010 ) or to DBMpaper (diazobenzyloxymethyl paper) by a modification of the procedure of SouTHERN (29) as previously described (14). DBM-paper was prepared from Whatman paper No. 540 according to ALWINE et al. (1) with the modification of $\mathrm{W}_{\mathrm{AHL}}$ et al. (35). The 1-[(m-nitrobenzyloxy)methyl] pyridinium chloride used in this procedure was kindly provided by professor $\mathrm{K}$. MARKER, Institute of Molecular Biology and Plant Physiology, University of Århus. Hybridization of labelled DNA to DNA immobilized on nitrocellulose filters was performed as described by DENHARDT (6) with the modifications of JEFFREYS and FlaVELL (15). Hybridization to DNA immobilized on DBM-paper was done in the presence of dextran sulfate according to WAHL et al. (35). Autoradiographic exposure was for 1-14 days with or without Kodak X-Omatic regular intensifying screens at $-80^{\circ} \mathrm{C}$.

\subsection{Containment}

Organisms containing in vitro recombinant DNA were handled under P1 conditions (9).

\section{RESULTS}

\subsection{Molecular analysis of the HIS4 region in C80-1253}

In a previous paper (25) the HIS4 region of the S. carlsbergensis chromosome III present in substitution strain C80-1253 was studied: Electropherograms of genomic DNA treated with EcoRI or HindIII were transferred to nitrocellulose filters according to SouTHERN (29) and subjected to hybridization with a ${ }^{32} \mathrm{P}$-labelled $9.4 \mathrm{~kb}$ PstI fragment from $S$. cerevisiae including the HIS4 gene. For both enzymes the substitution strain gave a band pattern that was different from, and weaker than, that of S. cerevisiae strain S288C.

In order to get further information on the differences between the HIS4 regions, the analysis was extended with restriction endonucleases BamHI, PstI, SalI, HpaI and Pvull. The autoradiogram is shown in Figure 2. The EcoRI digest of S288C included for comparison (lane 1) shows the S. cerevisiae HIS4 pattern previously described (13). The hybridization patterns obtained with PstI, SalI, HpaI and PvuII digest of C801253 (lanes $b$ through $\mathrm{e}$ ) are quite different from those of S288C (lanes $g$ through $\mathrm{k}$ ). Actually none of the enzymes produces a fragment which has the same size in the two strains. The BamHI 


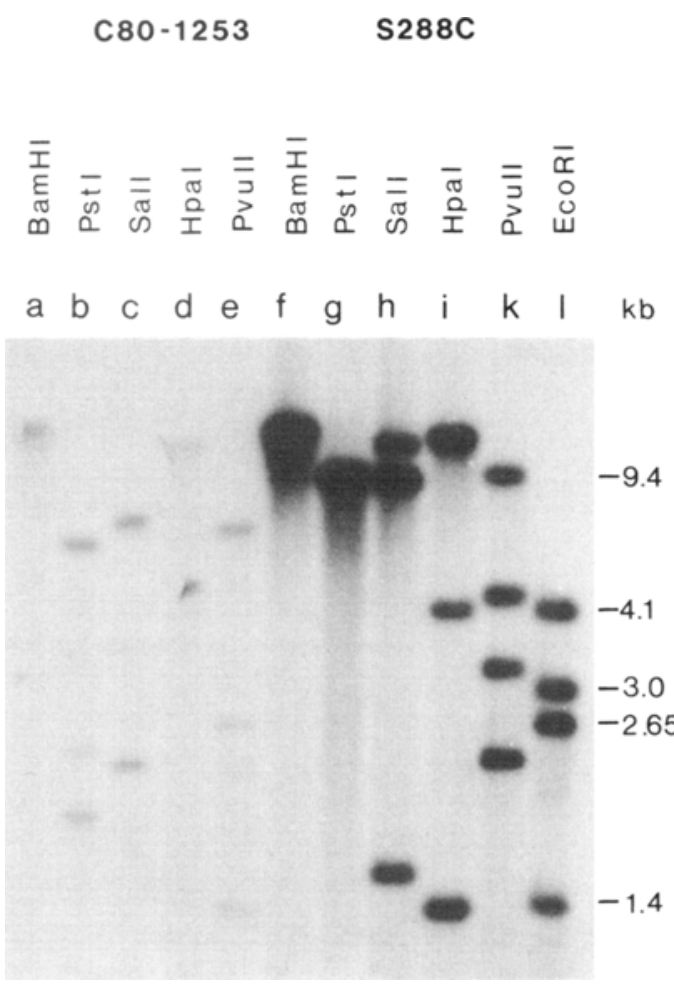

Figure 2. A comparison of the hybridization patterns in the HIS4 region of strains C80-1253 and S288C.

Two micrograms of DNA from the chromosome substitution strain $\mathrm{C} 80-1253$ and the $\mathrm{S}$. cerevisiae strain S288C were restricted, electrophoresed on a $0.7 \%$ agarose gel and transferred to a nitrocellulose filter. It was subjected to hybridization with a ${ }^{32} \mathrm{P}$ labelled preparation of the $9.4 \mathrm{~kb}$ Pstl fragment carrying the HIS4 gene from S. cerevisiae (14).

fragment seen in S288C (lane f) is about $20 \mathrm{~kb}$ and a similar sized fragment is obtained from C80-1253 (lane a). As in the previous study the $S$. carlsbergensis HIS4 region hybridizes with much lower efficiency to the radioactive probe than fragments from the corresponding $S$. cerevisiae regions. The results show that the nucleotide sequence in and around HIS4 of C80-1253 is different from that of $\mathrm{S} 288 \mathrm{C}$ at a large number of sites.

\subsection{Molecular analysis of the $L E U 2$ and $S U P$ - $R L 1$ regions in C80-1253}

Genetic analysis (25) revealed that the $S$. carlsbergensis chromosome III present in C80- $\mathrm{kb}$

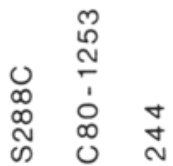

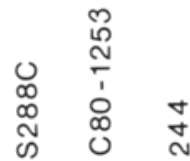

d e f

\section{$2.50-$ \\ $0.76-$}

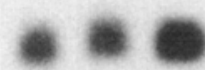

$0.30-$

Figure 3. Restriction endonuclease analysis of the $L E U 2$ and $S U P-R L I$ regions in the chromosome III substitution strain C80-1253.

DNA from strains S288C, C80-1253 and 244 was cleaved with Sall and Hhal, subjected to electrophoresis in $0.7 \%$ agarose and transferred to a nitrocellulose filter. The Sall digested DNA was hybridized to a $830 \mathrm{bp} \mathrm{EcoRI} \mathrm{-} \mathrm{Sall} \mathrm{fragment} \mathrm{con-}$ taining part of the LEU2 gene (5). The Hhal digested DNA was hybridized to a $1.1 \mathrm{~kb}$ BamHI EcoRI fragment mapping $1.6 \mathrm{~kb}$ from $S U P-R L I$ (26). Both fragments were from $S$. cerevisiae and in vitro labelled with ${ }^{32} \mathrm{P}$.

1253 does not recombine with S. cerevisiae chromosome III in the region his $4-M A T$ whereas normal levels of crossing over are seen in the re- 
gion $M A T-t h r 4$. HIS4 and LEU2 are both positioned in the non-recombining part while $S U P$ $R L I$ is located in the recombining part of the chromosome (cf. Figure 1).

To obtain information on the homology in the LEU2 region various restriction endonuclease digests of total DNA from strains C80-1253, S288C and 244 were subjected to agarose gel electrophoresis and transferred to a nitrocellulose filter according to Southern (29). The filter was incubated for hybridization with a ${ }^{32} \mathrm{P}$-labelled $830 \mathrm{bp} \mathrm{EcoRI} \mathrm{-} \mathrm{SalI} \mathrm{fragment} \mathrm{containing} \mathrm{part} \mathrm{of}$ the $L E U 2$ coding region (5). This fragment had been isolated from plasmid pYF91.2 (30). The hybridization pattern obtained with restriction endonuclease Sall is shown in Figure 3. In strain $\mathrm{S} 288 \mathrm{C}$ (lane a) a hybridizing band of $2.5 \mathrm{~kb}$ is present in agreement with previous observations $(7,30)$ whereas no fragments from $\mathrm{C} 80-1253$ (lane b) have sufficient homology to show hybridization to the probe. Similarly, no hybridization was seen to HindIII, BamHI, PstI and EcoRI digests of DNA from C80-1253. This result, together with the results described in section 3.1 above, shows that the nucleotide sequence in and around the genes HIS 4 and LEU2 positioned in the non-recombining part of chromosome III of C80-1253 is substantially different from the corresponding regions in $\mathrm{S}$. cerevisiae. There is one indication that the observed structural difference also determines a functional difference: The $L E U 2$ gene from $\mathrm{S}$. cerevisiae was originally cloned by complementation of the leuB mutation in E. coli (27). During attempts to clone genes from the $\mathrm{S}$. carlsbergensis chromosome III a gene pool in E. coli of BamHI cleaved C80-1253 DNA was prepared on $\mathrm{pC} 507$, a vector consisting of cosmid pJC75-58 and the TRPI - ARSI EcoRI fragment. DNA from this pool transforms S. cerevisiae leu2 to $\mathrm{Leu}^{+}$with high frequency, but fails to yield transformants of an $\mathrm{E}$. coli leuB strain upon simultaneous selection for ampicillin resistance and complementation of the leuB mutation. Although the structurally different $L E U 2$ genes can function as substitutes in yeast, only one of them appears to be able to substitute for the corresponding $\mathrm{E}$. coli gene.

The suppressor tRNA gene $S U P-R L I$ is located in the recombining part of chromosome III (25). Plasmid pPM6 contains a $1.1 \mathrm{~kb}$ BamHI -
EcoRI fragment mapping $1.6 \mathrm{~kb}$ from the SUP. $R L I$ gene (26). Plasmid pPM6 was used as a probe to various restriction digests of DNA from strains C80-1253, S288C and 244 by the technique of Southern (29). After cleavage of the DNA from strain S288C with Hhal two fragments of $760 \mathrm{bp}$ and $300 \mathrm{bp}$, respectively, hybridize to the probe (Figure 3, lane d). The same two fragments are seen to hybridize with the same intensity in the DNA digest of C80-1253 (lane e). When the DNA was digested with EcoRI, HindIII and BamHI the two strains gave likewise identical restriction fragment patterns, i.e. no difference in nucleotide sequence was detectable adjacent to $S U P-R L I$. Thus, among the regions investigated, only those which exhibit normal cross over frequencies have a close nucleotide sequence homology.

\subsection{Identification and analysis of $M A T$ and $H M$ loci in C80-1253}

The S. carlsbergensis chromosome III present in C80-1253 provides the cell with all essential functions normally provided by the $\mathrm{S}$. cerevisiae chromosome. It also contains $M A T$ and probably $H M L$ (25). Strathern et al. (33) had previously analysed the $M A T$ and $H M$ loci in S. carlsbergensis strain $\mathrm{CB} 11$ by restriction endonuclease mapping and an additional BglII site in $H M L \alpha$ was the only difference to $S$. cerevisiae detected. Prompted by the finding of sequence inhomologies in the HIS4 and LEU2 regions of the chromosomes III investigated, the degree of homology in the $M A T$ and $H M$ loci between strains C801253 and $\mathrm{S} 288 \mathrm{C}$ was examined.

DNA from strains S288C, C80-1253 and 244 was digested with restriction endonucleases and analysed by electrophoresis and SouTHERN blotting. In this experiment the probe was a ${ }^{32} \mathrm{P}$-labelled $4.2 \mathrm{~kb}$ EcoRI - HindIII fragment (M271) covering the entire $M A T \alpha$ gene from $\mathrm{S}$. cerevisiae. In Figure 4 lanes a-c, the autoradiogram of DNA digested with HindIII is shown. Strain S288C (lane a) exhibits the expected $(12,21)$ three bands: The $4.3 \mathrm{~kb}$ fragment originates from the $M A T$ locus, the $5.0 \mathrm{~kb}$ fragment from the $H M R$ locus and the $6.1 \mathrm{~kb}$ fragment from the $H M L$ locus. Three fragments with homology to the probe are also seen in C80-1253 (lane b), corresponding to the sizes $5.0 \mathrm{~kb}, 5.3 \mathrm{~kb}$ and 9.3 
kb. The $5.0 \mathrm{~kb}$ fragment hybridizes with the same intensity as the $H M R(5.0 \mathrm{~kb})$ band in lane $\mathrm{a}$, whereas the $5.3 \mathrm{~kb}$ and $9.3 \mathrm{~kb}$ fragments hybridize less strongly than the $M A T$ and $H M L$ bands of S288C. It is suggested that strain C80-1253 contains three mating type genes. The size and hybridization intensity of the $5.0 \mathrm{~kb}$ band may be taken to mean that this band covers the $H M R$ locus in C80-1253. Analogous results were obtained with BamHI, PstI and BglII digested DNA.

In order to assign the $M A T, H M L$ and $H M R$ loci to the three HindIII fragments three different probes were prepared by nick translation of isolated restriction fragments from cloned $\mathrm{S}$. cerevisiae DNA: (i) MHa2, a 1120 bp Hhal fragment from MAT $\alpha$ containing 438 bp of the $\mathrm{W}$ region; (ii) HP2, a 1500 bp HindIII - PstI fragment mapping about $500 \mathrm{bp}$ to the left of the $\mathrm{W}$ sequence of $H M L \alpha$; and (iii) MR2, a $530 \mathrm{bp}$ HindIII - EcoRI fragment mapping $300 \mathrm{bp}$ to the left of the W sequence of $M A T \alpha$. If the structural organization of the three mating type loci in $\mathrm{C} 80-1253$ is the same as in $\mathrm{S}$. cerevisiae the following predictions can be made: (i) The $\mathrm{MHa} 2$ probe should hybridize to the bands containing $H M L$ and $M A T$ due to the presence of $\mathrm{W}$ at both loci; (ii) the HP2 probe should hybridize to the $H M L$ bands; (iii) the MR2 probe to $M A T$; (iv) none of the probes should hybridize to $H M R$.

The results of such experiments are shown in Figure 4, lanes d-i. Thus, in lanes d-f, the SouthERN blots of HindIII digested DNA from strains S288C, C80-1253 and 244 were probed with the W specific MHa2 fragment. In S288C (lane d) strong hybridization is seen to $H M L(6.0 \mathrm{~kb})$ and $M A T(4.3 \mathrm{~kb})$. As expected, the probe has no homology to $H M R(5.0 \mathrm{~kb})$. In C80-1253 (lane e) the $5.3 \mathrm{~kb}$ and $9.3 \mathrm{~kb}$ fragments hybridize, but with weak homology. The same HindIII cleaved DNA probed with HP2 is shown in lanes g-i. The $H M L(6.1 \mathrm{~kb}$ ) band in $\mathrm{S} 288 \mathrm{C}$ (lane $\mathrm{g}$ ) hybridizes to HP2 while no hybridization is seen to $H M R$ and $M A T$. Instead, a $4.5 \mathrm{~kb}$ band shows up. The HP2 probe hybridizes weakly to the $9.3 \mathrm{~kb}$ fragment in C80-1253 (lane $\mathrm{h}$ ) and also here the 4.5 $\mathrm{kb}$ fragment is seen. No bands occur at the $5.0 \mathrm{~kb}$ and $5.3 \mathrm{~kb}$ positions. The $4.5 \mathrm{~kb}$ band in $\mathrm{S} 288 \mathrm{C}$ and $\mathrm{C} 80-1253$ can not be derived from chromosome III since it is absent in strain 244 (lane i) which contains the chromosome III transferred

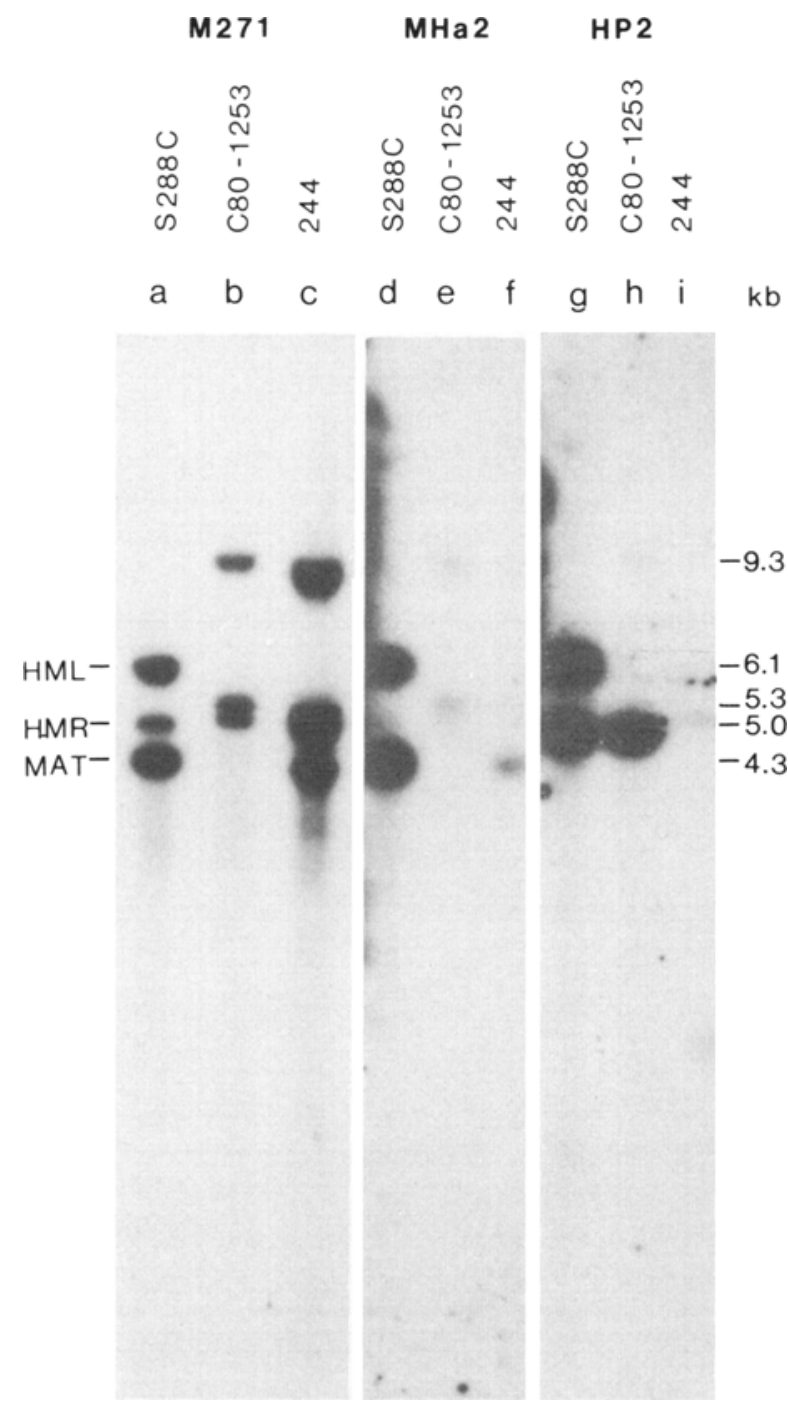

Figure 4. Identification of HindIII fragments carrying $M A T, H M L$ and $H M R$ of the chromosome substitution strain C80-1253.

DNA from strains S288C, C80-1253 and 244 was restricted, electrophoresed in $0.7 \%$ agarose and transferred to nitrocellulose filters. The filters were hybridized to three different ${ }^{32} \mathrm{P}$-labelled DNA preparations: M271, a $4.2 \mathrm{~kb}$ EcoRI - HindIII fragment covering the entire $M A T \alpha$ locus; $\mathrm{MHa} 2$, a $1120 \mathrm{bp}$ HhaI fragment derived from $M A T \alpha$ and containing 438 bp of the W region; and HP2, a 1500 bp HindIII - PstI fragment mapping about $500 \mathrm{bp}$ to the left of the W sequence of $H M L \alpha$. All probes had been cloned from S. cerevisiae (21). 
to $\mathrm{C} 80-1253$. That is, the HP2 probe contains a sequence located not only close to the left of $H M L$ but also elsewhere in the genome of S. cerevisiae. STRATHERN et al. (33) have made a similar observation with a BamHI - EcoRI fragment derived from the same region. When the MR2 probe was used only the MAT $\alpha$ band of S288C hybridized and in C80-1253 only the $5.3 \mathrm{~kb}$ HindIII fragment was seen.

In summary, $\mathrm{C} 80-1253$ contains three HindIII fragments of $9.3 \mathrm{~kb}, 5.3 \mathrm{~kb}$ and $5.0 \mathrm{~kb}$ showing homology to $M A T \alpha$ from $S$. cerevisiae (Figure 4, lane $b$ ): The $9.3 \mathrm{~kb}$ fragment hybridizes only to HP2 containing sequences close to the left of $H M L$ and to $\mathrm{MHa} 2$ with $\mathrm{W}$ sequences external to $M A T$. The $5.3 \mathrm{~kb}$ fragment hybridizes to $\mathrm{MHa} 2$ (W and sequences external to $M A T$ ) and to MR2 containing sequences to the left of $M A T$ whereas the $5.0 \mathrm{~kb}$ fragment does not hybridize to any of the three probes.

On the basis of these data I can assign the $H M L$ locus of $\mathrm{C} 80-1253$ to the $9.3 \mathrm{~kb}$ HindIII fragment, $M A T \mathrm{a}$ to the $5.3 \mathrm{~kb}$ HindIII fragment and $H M R$ to the $5.0 \mathrm{~kb}$ HindIII fragment. With this assignment, the predictions (i)-(iv) on the hybridization of the probes to the different bands are fulfilled, a fact which indicates that the three loci in $\mathrm{C} 80-1253$ are organized in the same way as in S. cerevisiae. Analogous experiments with the BamHI, PstI and BglII digested DNA confirmed this notion. In $H M L$, the conservation of sequences in $\mathrm{W}$ is as low as in sequences close to the left of W. An unstable and therefore presumably circular derivative of the $\mathrm{S}$. carlsbergensis chromosome III carrying HIS4 and LEU2 but lacking $T H R 4$ was found after a rare conversion of $M A T \mathrm{a}$ to $M A T \alpha$ (25). This indicates that the derivative has arisen by intrachromosomal recombination between $M A T$ and a $\mathrm{Y} \alpha$ containing cassette on the left arm distal to LEU2 and HIS4. Therefore, $H M L$ of C80-1253 identified by nucleotide sequence homology to $H M L$ of $\mathrm{S}$. cerevisiae is most likely located on the left arm of the $\mathrm{S}$. carlsbergensis chromosome III and contains $\alpha$ information. Also in MATa of C80-1253 the low degree of homology to $\mathrm{S}$. cerevisiae of $\mathrm{W}$ is continuing into the adjacent sequences to the left. In contrast, the sequences within and around the $H M R$ cassette have a high degree of homology to $\mathrm{S}$. cerevisiae. First, the homology to the $4.2 \mathrm{~kb}$
$M A T \alpha$ DNA is the same as in $\mathrm{S}$. cerevisiae as judged from hybridization intensities. Secondly, of the enzymes used in this analysis only BglII reveals a difference between the two strains while HindIII, BamHI and PstI produce identical patterns.

As described above, strong hybridization was obtained using the $4.2 \mathrm{~kb} M A T \alpha$ DNA as a probe while the probes $\mathrm{MHa} 2, \mathrm{HP} 2$ and MR2 gave low intensity bands. This strongly indicates that the homology between the three loci of C80-1253 and the $M A T \alpha$ probe resides within the $\mathrm{X}, \mathrm{Y}$ and $Z$ regions, a notion which was further tested in experiments presented in section 3.5 below.

\subsection{Does strain 244 contain two structurally different chromosomes III?}

Previously it was found that strain 244 is structurally heteroallelic in the HIS4 region (25). The one allele, which is also present in C80-1253, is substantially different from the S. cervisiae HIS4 region (ref. 13 and section 3.1 of the present paper) while the other is similar, although possibly not identical to $S$. cerevisiae. Therefore DNA from strain 244 was included in the molecular analyses described above in sections 3.2 and 3.3 and the results were as follows: $S$. cerevisiae LEU2 DNA did not hybridize to DNA from strain 244 (Figure 3, lane c) just as it did not to C80-1253 DNA. Likewise, strain 244 gave the same hybridization pattern as $\mathrm{C} 80-1253$ when probed with SUP-RLI DNA (Figure 3, lane f). The analysis of the $M A T$ and $H M$ loci showed, in addition to the fragments hybridizing in C801253 , one band of the size $4.3 \mathrm{~kb}$ (Figure 4, lane c). This $4.3 \mathrm{~kb}$ HindIII fragment was found to carry $M A T$ (Figure 4, lanes $\mathrm{f}$ and i). With all enzymes tested (HindIII, BamHI, PstI and BgliI) the fragments carrying this $M A T$ allele had sizes identical to $S$. cerevisiae although they hybridized less. Until now, only chromosomes III carrying the HIS4 allele present in C80-1253 have been isolated in a $\mathrm{S}$. cerevisiae background. The data presented here show that strain 244 is structurally heterozygous not only in and around the HIS4 gene, but also in the regions adjacent to $M A T$. The absence of a $6.0 \mathrm{~kb}$ fragment hybridizing to the M271 probe (Figure 4, lane c) reveals that strain 244 does not contain a $H M L$ region identical to that of S. cerevisiae. Whether strain 
244 is structurally heterozygous for the regions containing LEU2, SUP-RLI, HML and HMR can not be determined at this point. In contrast to the two HIS4 alleles, strain 244 certainly does not contain an allele of $L E U 2$ that is closely related in sequence to $L E U 2$ of $\mathrm{S}$. cerevisiae.

\subsection{Strong conservation of sequences in the cassettes at the HML, MAT and HMR loci}

The experiments described in section 3.3 identified three loci in C80-1253 corresponding to the $H M L, M A T$ and $H M R$ loci of S. cerevisiae. A strong homology of the cassettes was also indicated.

In order to study this sequence conservation further the structure of the three loci in C80-1253 was examined using restriction enzymes that cut the DNA frequently. The complete nucleotide sequences of $H M L \alpha$ and $M A T \mathrm{a}$ from S. cerevisiae have been determined (2). Using this information two probes were isolated from the $4.3 \mathrm{~kb}$ MAT $\alpha$ HindIII fragment: (i) $\mathrm{MHa3}$, a $948 \mathrm{bp}$ HhaI fragment bounded by coordinates 1226 and 2174 and consisting of $747 \mathrm{bp}$ of $\mathrm{Y} \alpha$ and $201 \mathrm{bp}$ of $X$ and (ii) MHil, a 882 bp Hinfl fragment bounded by coordinates 129 and 1011 and consisting of $594 \mathrm{bp}$ of $\mathrm{W}$ and $288 \mathrm{bp}$ of $\mathrm{X}$. The presence or absence in C80-1253 of restriction sites predicted from the published $\mathrm{S}$. cerevisiae sequences (2) was examined using these two probes.

Total DNA from strains S288C and C80-1253 was digested with restriction endonucleases DdeI, TaqI, AluI, HaeIII, Hhal and HinfI, subjected to electrophoresis in $2 \%$ agarose gels and transferred to diazobenzyloxymethyl-paper. A plasmid consisting of pBR322 with a $5.0 \mathrm{~kb}$ HindIII insert carrying HMRa from strain XP810B $(21,23)$ was included for comparison. The electropherograms were hybridized with ${ }^{32} \mathrm{P}$-labelled DNA consisting of either the $\mathrm{MHa} 3$ or the MHil probes described above. Figure 5 shows the result when DNA samples cleaved with DdeI, TaqI, AluI and HaellI were probed with MHa3. Table I gives the sizes of all restriction fragments hybridizing including data for the other probe and enzymes.

The interpretation of the results is based on the following assumptions: (i) Restriction fragments having the same size are taken to be homologous; (ii) when the sizes of two fragments in one strain can be added up to the size of one fragment in the other they are taken to be homologous unless there is evidence to the contrary; (iii) there is identity between corresponding segments of the three loci, as has been shown in S. cerevisiae $(21,33)$. All results could be interpreted on the basis of the two first assumptions and they were consistent with the last assumption. Furthermore, when a new restriction site is postulated in C80-1253 a single base pair change in the $\mathrm{S}$. cerevisiae sequence could give rise to the restriction site in all cases.

As an example of the analysis I shall point to the TaqI digested S288C ( $M A T \alpha$ ) DNA probed with $\mathrm{MHa} 3$ and shown in Figure 5, lane d. Four bands of $1174 \mathrm{bp}, 730 \mathrm{bp}, 325 \mathrm{bp}$ and $122 \mathrm{bp}$ hybridize to the probe. From the $H M L \alpha$ and $M A T \alpha$ sequence (2) it can be seen that the 730 bp and 122 bp fragments are generated from the TaqI sites at positions 1042, 1775 and 1897; TaqI sites at positions 1999 and 2324 give the $325 \mathrm{bp}$ fragment. Two bands of $60 \mathrm{bp}$ and $42 \mathrm{bp}$ (coordinates 1897, 1939 and 1999) from $H M L \alpha$ and $M A T \alpha$, also expected to hybridize to the probe, are too small to be detected. The $M A T$ a sequence (2) predicts a TaqI fragment of $1174 \mathrm{bp}$ (coordinates 1042 and 2219 ), but since $\mathrm{S} 288 \mathrm{C}$ is $M A T \alpha$ the observed $1174 \mathrm{bp}$ fragment must be derived from $H M R \mathbf{a}$, demonstrating that the TaqI site at position 1042 also is present in X of HMRa. Likewise in $H M R \mathbf{a}$ DNA cloned from $\mathrm{S}$. cerevisiae (lane $\mathrm{f}$ ) the $1174 \mathrm{bp}$ band is seen, confirming that this fragment in $\mathrm{S} 288 \mathrm{C}$ is derived from HMRa. DNA from C80-1253 (MATa) cleaved with TaqI (lane e) shows three bands of $1174 \mathrm{bp}, 670 \mathrm{bp}$ and $585 \mathrm{bp}$. The presence of the $1174 \mathrm{bp}$ band shows that the TaqI sites at positions 1042 and 2219 are conserved in HMRa of C80-1253 and consistent with the data it is assumed that these sites are also present in $M A T \mathrm{a}$ and $H M L \alpha$. The $670 \mathrm{bp}$ and $585 \mathrm{bp}$ fragments are then derived from $H M L \alpha$ and are explained as follows (see also Figure 6): In S288C five fragments are derived from four TaqI sites between positions 1042 and 2324; in C80-1253 only two fragments occur. The presence in $H M L \alpha$ of the TaqI fragments of 670 $\mathrm{bp}$ and $585 \mathrm{bp}$ is interpreted as loss of the four sites in $\mathrm{Y} \alpha$ in $\mathrm{C} 80-1253$, when compared to $\mathrm{S} 288 \mathrm{C}$, and the occurrence of one new site either 
around position 1630 or 1720 . From the DNA sequence of $H M L \alpha$ a single transition at 1728 from AT to GC will generate the TaqI sequence TCGA leading to the two fragments of $686 \mathrm{bp}$ and 596 bp seen in lane e. In summary, $H M L \alpha$ in C80-1253 differs from $H M L \alpha$ in S288C by the loss of four TaqI sites in Y $\alpha$ at position 1775, 1897,1939 and 1999 and the presence of a site at 1728. The TaqI sites located in X (position 1042) and $\mathrm{Zl}$ (position 2324 in $H M L \alpha$ and $M A T \alpha$; 2219 in MATa and $H M R \mathbf{a}$ ) are present at all three loci in both strains.

Analogous analyses of the data presented in Figure 5 and Table I lead to the restriction maps of $H M L \alpha$, MATa and HMRa in C80-1253 depicted in Figure 6 and summarized in Figure 7. It is seen that in $\mathrm{W}$ four of the six restriction sites present in S288C were not found in C80-1253. In $\mathrm{X}$ all nine sites tested for were present in $\mathrm{C80}$ 1253. The $\mathrm{Ya}$ sequence of $H M L \alpha$ in $\mathrm{C} 80-1253$ has only retained one site out of eleven tested for while a new TaqI and a new AluI site were found at coordinates 1728 and 2153 , respectively. Of the three sites investigated in $\mathrm{Zl}$, one turned out to be missing. All data obtained are consistent with the assumption that corresponding segments in the two or three loci of the S. carlsbergensis chromosome are in fact identical.

Thus, with regard to the analyzed S. cerevisiae and $\mathrm{S}$. carlsbergensis chromosomes, a high degree of conservation exists for the cassettes of the $M A T$ and $H M$ loci. Although the results presented in Figure 7 indicate that the degree of diversity in $\mathrm{W}$ and $\mathrm{Ya}$ is similar, hybridization intensities (Figures 4 and 5) show that conservation in $\mathrm{Y} \alpha$ is considerably stronger than in W. Although all restriction sites tested for in the $\alpha$ l coding sequence have changed, this sequence is more conserved than the coding sequence of HIS4 and LEU2 as judged by hybridization intensities. Finally, it should be noted that inside the cassettes the strongest conservation is seen in $X$ and $Z 1$, sequences involved in transposition of $\mathrm{Y}$.

\section{DISCUSSION}

The S. carlsbergensis chromosome III present in C80-1253 has a close functional homology to the wild type $S$. cerevisiae chromosome III since it provides the cell with all essential functions normally provided by the $\mathrm{S}$. cerevisiae chromo- some. Furthermore, it contains wild type equivalents to all genes of the latter that have been looked for. Recombination in the HIS4 - MAT region does not occur between the $S$. carlsbergensis chromosome and the $\mathrm{S}$. cerevisiae chromosome whereas a normal level of recombination is seen in the MAT - THR4 region (25). Molecular analyses using cloned $\mathrm{S}$. cerevisiae DNA both from the non-recombining region (HIS4 and $L E U 2$, Figures 2 and 3 ) and the recombining region (SUP-RLI, Figure 3) revealed that the absence of crossing over is correlated with extensive DNA sequence inhomology.

The inhomology is particularly pronounced at $L E U 2$, where no hybridization was detected between the $S$. carlsbergensis chromosome and the S. cerevisiae probe. Hybridization conditions with low stringency (e.g. by lowering the temperature) might very well allow the technique to reveal a $\mathrm{S}$. carlsbergensis band pattern.

The presence in C80-1253 of three loci corresponding to $H M L, M A T$ and $H M R$ of S. cerevisiae was shown by molecular hybridization using DNA fragments containing sequences inside and outside the cassettes of MAT $\alpha$ and $H M L \alpha$ from S. cerevisiae. These experiments also showed that weak homology exists to the left of $\mathrm{W}$ of $H M L$ and $M A T$. That the molecularly identified $\mathrm{S}$. carlsbergensis $H M L \alpha$ locus is indeed positioned on the left arm of chromosome III, distal to HIS4 and $L E U 2$, is indicated by the isolation of a mitotically unstable derivative of the $S$. carlsbergensis chromosome III carrying HIS4 and LEU2, but lacking $T H R 4$ after a rare conversion of $M A T \mathrm{a}$ to $M A T \alpha(25)$. In analogy to the findings by STrathern et al. (32) in S. cerevisiae, we suppose that the derivative is circular. Regarding the location of $H M R \mathbf{a}$, a molecular analysis of spores which have recombined in the $M A T-T H R 4$ region indicates that $H M R \mathbf{a}$ is on the right arm distal to $M A T$. The same unpublished data also confirmed that the sequences immediately outside $H M R \mathbf{a}$ are closely homologous to S. cerevisiae. A generalization of these findings would be that the gene order on the S. carlsbergensis chromosome is the same as in S. cerevisiae.

Taken together, genetic and molecular analyses reveal that the chromosome III in C80-1253 is composed of a part closely homologous to the $S$. cerevisiae chromosome III and another part 
which has extensive nucleotide sequence diversity when compared to the latter. The border between the two parts seems to be located close to the right of $M A T$. It is tempting to speculate that this chromosome has been generated by crossing over in the $M A T$ locus, between two homeologous chromosomes.

The detailed analysis of the $M A T$ and $H M$ loci in $\mathrm{C} 80-1253$ showed varying sequence homology to $\mathrm{S}$. cerevisiae in the different segments. In W homology was low whereas almost complete homology was found in $\mathrm{X}$. In $\mathrm{Y} \alpha$ many restriction site differences were seen in the $\alpha$ l coding sequence, but rather strong hybridization intensities were obtained. The strong conservation of sequences in the $\mathrm{X}$ segment could be explained in several ways. An asymmetric gene conversion model for mating type change has been proposed (17) and it has been shown that some intrachromosomal pairing, at least in the $\mathrm{X}$ and $\mathrm{Zl}$ regions, takes place during switching $(10,17)$. Thus, sequences in $\mathrm{X}$ are involved in the transposition event. The high degree of conservation of sequences in $\mathrm{X}$ observed here might reflect very specific requirements on $X$ either in the pairing or as a substrate for the enzymes effecting the transposition involved. Alternatively, requirements for conservation of gene products may be considered. Two transcripts of the $\mathrm{X}$ region have been identified $(18,23)$. The $\alpha 2$ transcript is initiated within a small segment of the $\mathrm{Y} \alpha$ region and the a 2 transcript within the $\mathrm{X}$ region. The two transcripts terminate at the same nucleotide (22).

The switching of mating type is a directed process. That is, in a cells transposition from $H M L$ is favored while in $\alpha$ cells transposition from $H M R$ is favored $(19,31)$. It has been suggested that the presence of $\mathrm{W}$ and $\mathrm{Z} 2$ at $H M L$ and their absence at $H M R$ play a role in the recognition of $H M L$ and $H M R$ during switching (19). Interestingly the $\mathrm{W}$ sequence in C80-1253 appears to be identical in $H M L$ and $M A T$, although being different from $\mathrm{W}$ in $\mathrm{S}$. cerevisiae. This supports the idea of a functional role of $\mathrm{W}$ in mating type interconversion.

The chromosome III analysed in this paper is derived from strain 244 and it has one of the two HIS4 alleles present in strain 244 (25). It was speculated whether the other, more S. cerevisiae- like, HIS4 allele is located on a chromosome III which will show normal recombination levels in S. cerevisiae (25). The present analysis of strain 244 shows that it is heterozygous not only for the $H I S 4$ region, but also for sequences closely linked to $M A T$. With respect to the $L E U 2, S U P-R L I$ and $H M L$ regions no difference is seen between strain 244 and C80-1253: It is concluded that strain 244 does not contain a chromosome III that has close nucleotide sequence homology to a standard S. cerevisiae chromosome III in all regions.

\section{ACKNOWLEDGEMENTS}

I gratefully acknowledge the receipt of plasmids from Drs. J. Friesen, M. Olson and K. Tatchell. I wish to thank M. C. KIEllandBrandt, T. Nilsson-Tillgren, J. G. L. PeterSen, C. Poulsen and C. Guermansen for advice and fruitful discussions, D. voN WETTSTEIN for support and encouragement and G. BANK for excellent technical assistance. This work was supported by grants $16-1740$.H-547 and 16-3113.H605 from the Danish National Technical Science Research Council to D. voN WETTSTEIN.

\section{REFERENCES}

1. Alwine, J. C., D. J. Kemp \& G. R. Stark: Method for detection of specific RNAs in agarose gels by transfer to diazobenzyloxymethylpaper and hybridization with DNA probes. Proc. Nat. Acad. Sci. USA 74, 5350-5354 (1977)

2. Astell, C. R., L. Ahlstrom-Jonasson, M. Smith, K. Tatchell, K. A. Nasmyth \& B. D. HALL: The sequence of the DNAs coding for the mating-type loci of Saccharomyces cerevisiae. Cell 27, 15-23 (1981)

3. Birnboim, H. C. \& J. Doly: A rapid alkaline extraction procedure for screening recombinant plasmid DNA. Nucl. Acids Res. 7, 15131523, (1979)

4. Brandriss, M. C., J. W. Stewart, F. Sherman \& D. BotsTEIN: Substitution of serine caused by a recessive lethal suppressor in yeast. J. Mol. Biol. 102, 467-476 (1976)

5. Chinault, A. C. \& J. Carbon: Overlap hybridization screening: Isolation and characterization of overlapping DNA fragments surrounding the leu 2 gene on yeast chromosome III. Gene 5, 111-126 (1979) 
6. Denhardt, D. T:: A membrane-filter technique for the detection of complementary DNA. Biochem. Biophys. Res. Commun. 23, 641-646 (1966)

7. Dobson, M. J., S. M. Kingsman \& A. J. KINGSMAN: Sequence variation in the $L E U 2$ region of the Saccharomyces cerevisiae genome. Gene 16, 133-139 (1981)

8. Dutcher, S. K.: Internuclear transfer of genetic information in karl-l/KARI heterokaryons in Saccharomyces cerevisiae. Mol. Cell. Biol. 1, 245-253 (1981)

9. Guidelines for research involving recombinant DNA molecules. Federal Register U. S. 47 (77), 17180-17198 (1982)

10. Haber, J. E., D. W. Mascioli \& D. T. Rogers: Illegal transposition of mating-type genes in yeast. Cell 20, 519-528 (1980)

11. Herskowitz, I. \& Y. Oshima: Control of cell type in Saccharomyces cerevisiae: Matingtype and mating-type interconversion. In: The Molecular Biology of the Yeast Saccharomyces. J. N. Strathern, E. W. Jones and J. R. Broach, eds. Cold Spring Harbor, New York: Cold Spring Harbor Laboratory Press, pp 181-209 (1981)

12. Hicks, J., J. N. Strathern \& A. J. S. Klar: Transposable mating type genes in Saccharomyces cerevisiae. Nature 282, 478-483 (1979)

13. Holmberg, S., M. C. Kielland-Brandt, T. Nilsson-Tillgren \& J. G. L. Petersen: Molecular characterization of three his 4 deletion mutants in Saccharomyces cerevisiae. Carlsberg Res. Commun. 44, 283-288 (1979)

14. Holmberg, S., J. G. L. Petersen, T. NilssonTillgren \& M. C. Kielland-Brandt: Molecular characterization of a Saccharomyces plasmid containing the HIS4 gene. Carlsberg Res. Commun. 44, 269-282 (1979)

15. Jeffreys, A. J. \& R. A. Flavell: A physical map of the DNA regions flanking the rabbit $\beta$ globin gene. Cell 12, 429-439 (1977)

16. Kielland-Brandt, M. C., T. Nilsson-Tillgren, S. Holmberg, J. G. L. Petersen \& B. A. SVENNINGSEN: Transformation of yeast without the use of foreign DNA. Carlsberg Res. Commun. 44, 77-87 (1979)

17. Klar, A. J. S., J. McIndoo, J. N. Strathern \& J. B. Hicks: Evidence for a physical interaction between the transposed and the substituted sequences during mating type gene transposition in yeast. Cell 22, 291-298 (1980)

18. Klar, A. J. S., J. N. Strathern, J. R. Broach \& J. B. HICKs: Regulation of transcription in expressed and unexpressed mating type cassettes of yeast. Nature 289, 239-244 (1981)
19. Klar, A. J. S., J. B. Hicks \& J. N. Strathern: Directionality of yeast mating-type interconversion. Cell 28, 551-561 (1982)

20. Mortimer, R. K. \& D. SCHILD: Genetic map of Saccharomyces cerevisiae. Microbiol. Rev. 44, 519-571 (1980)

21. Nasmyth, K. A. \& K. Tatchell: The structure of transposable yeast mating type loci. Cell 19, 753-764 (1980)

22. Nasmyth, K. A., K. Tatchell, B. D. Hall, C. Astell \& M. SMith: Physical analysis of mating-type loci in Saccharomyces cerevisiae. Cold Spring Harbor Symp. Quant. Biol. 45, 961-981 (1981)

23. Nasmyth, K. A., K. Tatchell, B. D. Hall, C. Astell \& M. SMith: A position effect in the control of transcription at yeast mating type loci. Nature 289, 244-250 (1981)

24. Nilsson-Tillgren, T., J. G. L. Petersen, S. Holmberg \& M. C. Kielland-Brandt: Transfer of chromosome III during kar mediated cytoduction in yeast. Carlsberg Res. Commun. 45, 113-117 (1980)

25. Nilsson-Tillgren, T., C. Guermansen, M. C. Kielland-Brandt, J. G. L. Petersen \& S. Holmberg: Genetic differences between Saccharomyces carlsbergensis and $\mathrm{S}$. cerevisiae. Analysis of chromosome III by single chromosome transfer. Carlsberg Res. Commun. 46, 65-76 (1981)

26. Olson, M. V., G. S. Page, A. Sentenac, P. W. Piper, M. Worthington, R. B. Weiss \& B. D. HALL: Only one of two closely related yeast suppressor tRNA genes contains an intervening sequence. Nature 291, 464-469 (1981)

27. Ratzkin, B. \& J. Carbon: Functional expression of cloned yeast DNA in Echerichia coli. Proc. Nat. Acad. Sci. USA 74, 487-491 (1977)

28. Rigby, P. W. J., M. Dieckmann, C. Rhodes \& P. BERG: Labeling deoxyribonucleic acid to high specific activity in vitro by nick translation with DNA polymerase I. J. Mol. Biol. 113, 237251 (1977)

29. Southern, E. M.: Detection of specific sequences among DNA fragments separated by gel electrophoresis. J. Mol. Biol. 98, 503-517 (1975)

30. Storms, R. K., J. B. McNeil, P. S. KhandeKAR, G. AN, J. PARKer \& J. D. Friesen: Chimeric plasmids for cloning of deoxyribonucleic acid sequences in Saccharomyces cerevisiae. $\mathrm{J}$. Bacteriol. 140, 73-82 (1979)

31. Strathern, J. N. \& I. Herskowitz: Asymmetry and directionality in production of new cell types during clonal growth: the switching pat- 
tern of homothallic yeast. Cell 17, 371-381 (1979)

32. Strathern, J. N., C. S. Newlon, I. HerskowiTz \& J. B. Hicks: Isolation of a circular derivative of yeast chromosome III: Implications for the mechanism of mating type interconversion. Cell 18, 309-319 (1979)

33. Strathern, J. N., E. Spatola, C. McGill \& J. B. Hicks: Structure and organization of transposable mating type cassettes in Saccharomyces yeasts. Proc. Nat. Acad. Sci. USA 77, 2839-2843 (1980)

34. SutCLIFFE, J. G.: pBR322 restriction map deri- ved from the DNA sequence: accurate DNA size markers up to 4361 nucleotide pairs long. Nucl. Acids Res. 5, 2721-2728 (1978)

35. Wahl, G. M., M. Stern \& G. R. Stark: Efficient transfer of large DNA fragments from agarose gels to diazobenzyloxymethyl-paper and rapid hybridization by using dextran sulfate. Proc. Nat. Acad. Sci. USA 76, 3683-3687 (1979)

36. YANG, R. C-A., J. LIS \& R. Wu: Elution of DNA from agarose gels after electrophoresis. In: Methods in Enzymology, R. Wu ed. Academic Press, New York, 68, 176-182 (1979) 